\title{
Hochwasserbewusstsein 10 Jahre nach dem „Jahrhundertereignis" im Osterzgebirge und an der Elbe
}

\begin{abstract}
In den 10 Jahren, die seit dem Extremhochwasser in Sachsen vergangen sind, wurde viel für den Hochwasserschutz getan: die Vorhersage, die Kommunikation sowie die Hochwasserschutzanlagen wurden verbessert und es wurden neue Deiche sowie Hochwasserrückhaltebecken errichtet oder angepasst. Eine wichtige Frage ist aber, wie sich das Hochwasserbewusstsein der potenziell betroffenen Bevölkerung entwickelt hat. Im Beitrag wird der Frage nachgegangen, ob die Lehren von 2002 in Erinnerung sind und weitergegeben wurden oder ob das Hochwasserbewusstsein nachgelassen hat und man sich angesichts besseren Schutzes in Sicherheit wiegt.
\end{abstract}

\section{Einführung}

Im Jahre 2012 jährt sich zum 10. Male das Hochwasser im Osterzgebirge und an der Elbe. Dieses Ereignis, welches seinerzeit auch die Vorstellungskraft sehr vieler Fachleute überstieg, hat zahlreiche Aktivitäten im Bereich der Nachsorge und der Vorsorge für künftige Ereignisse nach sich gezogen. Zur Nachsorge gehören die Schadensbeseitigung, die Ereignisanalyse und der Wiederaufbau, welcher den erkannten Gefährdungen Rechnung tragen soll. Im Rahmen der Vorsorge spielen die Flächenvorsorge zur Erhöhung des natürlichen Hochwasserrückhaltes, der gemeinschaftliche und individuelle technische Hochwasserschutz sowie der organisatorische Hochwasserschutz mit Risiko-, Informations- und Verhaltensvorsorge eine wichtige Rolle. Diese Maßnahmen haben die Aufgabe, die Verletzbarkeit (Vulnerability) der Menschen, ihrer Siedlungen, Infrastruktur und Produktionsanlagen durch das Naturereignis Hochwasser zu vermindern und deren Widerstandskraft (Resilience) zu erhöhen. Während Überflutungsbereiche und Schäden quantifiziert werden können, ist die Quantifizierung von Vulnerability und Resilience sowie des zur organisatorischen Vorsorge besonders notwendigen Hochwasserbewusstseins nach wie vor schwierig.

\section{Hochwasserbewusstsein}

Der Ausgleich zwischen dem Überfluss und dem Mangel an Wasser ist eine der wesentlichen Aufgaben der Wasserwirtschaft und des Wasserbaus. Hochwasser und Trockenheit können räumlich und zeitlich variieren. Klimaprojektionen gehen momentan davon aus, dass $z$. B. in der Heimatregion der Verfasser die Sommer trockener und die Winter feuchter sowie schneeärmer werden. Allgemein wird vermutet, dass Extreme zunehmen, was im Hochwasserfall zu kürzeren Konzentrationszeiten und höheren Scheiteln führen kann.

In der Geschichte haben Hochwasser und Trockenheit immer wieder das Leben der Menschen beeinflusst, ihren Alltag unterbrochen, verändert oder sogar ihre Existenz bedroht. Trotzdem zog es die Menschen immer wieder an die Flüsse, vor allem, wenn die Wiederkehrintervalle der Ereignisse länger als eine Generation waren und die Erinnerung oder Überlieferung des letzten Hochwasserereignisses verblasst waren. Am Fluss gab und gibt es fruchtbares Ackerland. Die Flächen sind leicht zu besiedeln. Flüsse dienen als Verkehrswege und in den Tälern bieten sich für Straßen und Bahnen gute Trassierungsmöglichkeiten. Vor allem sind Flüsse auch Wasser- und Energielieferanten, die schon sehr früh für Wassermühlen und später für Wasserkraftwerke genutzt wurden.

Dass es außerordentliche Ereignisse gab, gibt und geben wird, für die ein zuverlässiger Schutz nicht oder nicht mit vertretbaren Mitteln erreicht werden kann, ist eine schmerzliche Erfahrung. Beispiele dafür aus dem Elbeinzugsgebiet sind die Hochwasser im Osterzgebirge in den Jahren 1897, 1927, 1957, 1958, 1981 und 2002. Sie wurden durch die Großwetterlage $\mathrm{Vb}$ ausgelöst, bei der feuchte Luft vom Atlantik über das Mittelmeer das Erzgebirge von Süden erreichte, um dort abzuregnen, was den Erzgebirgskamm zu einem besonderen Hochwasserentstehungsgebiet macht.

Nach dem Hochwasser ist vor dem Hochwasser - so lautet eine alte Erkenntnis, die den Kreislauf von Hochwasservorsorge, Hochwasserereignis, Hochwasserbewältigung, Hochwassernachsorge und hochwasserfreier Periode prägnant beschreibt. Die Bereitschaft zur Bereitstellung von Sach- und Personalmitteln für den Hochwasserschutz durch die Öffentlichkeit (Politik, Verwaltung) und Privatpersonen hängt immer vom so genannten Hochwasserbewusstsein bzw. der jeweiligen Wahrnehmung der Hochwassergefahr ab. Leider nimmt dieses erfahrungsgemäß nach einem Ereignis relativ schnell ab und wird durch andere Ereignisse oder Probleme in Anspruch 
genommen, wobei sicherlich zwischen Verantwortungsträgern, Fachleuten, Betroffenen und Nicht-Betroffenen zu unterscheiden ist. Die diesbezüglichen Aufmerksamkeitskurven für die Region, auf Landesebene und für Fachleute in Bild 1 beruhen auf Annahmen und könnten durch die Einbeziehung soziologischer Untersuchungen auf verschiedenen Skalen zweifellos noch verfeinert und genauer quantifiziert werden, wenngleich es schwierig sein dürfte, eine geeignete Maßeinheit zu finden. Jedoch sollen mit dieser vereinfachten Darstellung einige Tendenzen veranschaulicht werden:

1. Die gesellschaftliche Wahrnehmung im Allgemeinen nimmt relativ schnell wieder ab. Ob der „Vergessenszyklus“ sich an biblische Zeitperioden von 7 oder 12 Jahren anlehnt oder mit einer Paretoverteilung oder Paretos 80:20-Prinzip [2] beschrieben werden kann, soll hier nicht weiter betrachtet werden.

2. Durch die Entwicklung der Medien nimmt die kurzzeitige überregionale Wahrnehmung zu.

3. Aufgrund des zweimaligen Abstandes der Hochwasserereignisse im Erzgebirge von 30 Jahren rechneten einige im Jahre 1987 mit einem vergleichbaren Hochwasser wie 1897, 1927 und 1957, das aber ausblieb. Es konnte auch kein Anhaltspunkt für eine solche Periodizität gefunden werden.
4. Nach Hochwasserereignissen wurden planerische, gesetzgeberische, organisatorische und bautechnische Initiativen des Hochwasserschutzes getroffen, wie in Bild 1 gezeigt wird. Da deren Vorbereitung und Umsetzung stets eine gewisse Weile in Anspruch nahm, ist ein zeitlicher Nachlauf dieser Aktivitäten zu beobachten.

Die Kurven unterstellen, dass das allgemeine regionale Hochwasserbewusstsein in weniger als 10 Jahren auf die Hälfte des Wertes unmittelbar nach dem Ereignis gesunken ist. Dabei lässt die Darstellung dieser „Halbwertszeit“ offen, ob zum Beispiel bei „50 \% Hochwasserbewusstsein" bei einem (jedem) Individuum die Aufmerksamkeit auf $50 \%$ zurückgegangen ist oder ob bei der Hälfte der Individuen die Hochwasseraufmerksamkeit unter einen Schwellwert gefallen ist, der zu gering ist, um diese Personengruppe z. B. für Vorsorgemaßnahmen zu gewinnen.

Eine solche Betrachtung lässt die Frage nach einer möglichen quantitativen Beschreibung oder „Messung“ des Hochwasserbewusstseins aufkommen. Da es sicher schwierig ist, hier eine geeignete und allgemeingültige Definition oder Formel zu finden, wurde versucht, für eine quantitative Beschreibung hier auf die Interpretation von Befragungsergebnissen zurückzugreifen.

\section{Umfrage}

Um den aktuellen Stand des Hochwasserbewusstseins in einem typischen vom Sommerhochwasser 2002 betroffenen Einzugsgebiet näher zu betrachten, wurde von den Autoren unter Mitwirkung von Studentinnen der Fachrichtung Hydrologie der TU Dresden eine Umfrage im Einzugs- und Überflutungsgebiet der Weißeritz und der Elbe durchgeführt. Die verwendeten Fragebögen waren im Rahmen des ERA-Net-CRUE-Projektes „Sustainable Strategies of Urban Flood Risk Management with non-structural Measures to cope with the Residual Risk " SUFRI (Nachhaltige Strategien für das Hochwasserschutzmanagement in Städten zur Beherrschung des Restrisikos mit nicht technischen Maßnahmen - www.sufri.tugraz. at) in Zusammenarbeit mit dem Institut für Wasserbau und Wasserwirtschaft der TU Graz dem Institut für Soziologie, Abteilung für Krisen- und Katastrophenforschung der Karl-Franzens-Universität Graz und drei weiterer Partner aus Italien sowie Spanien ausgearbeitet und entsprechend an die Gegebenheiten der Untersuchungsgebiete angepasst worden.

\subsection{Untersuchungsgebiet}

Während des Hochwassers 2002 wurde ein Teil des Stadtgebietes von Dresden zunächst am 12. und 13. August 2002 von der

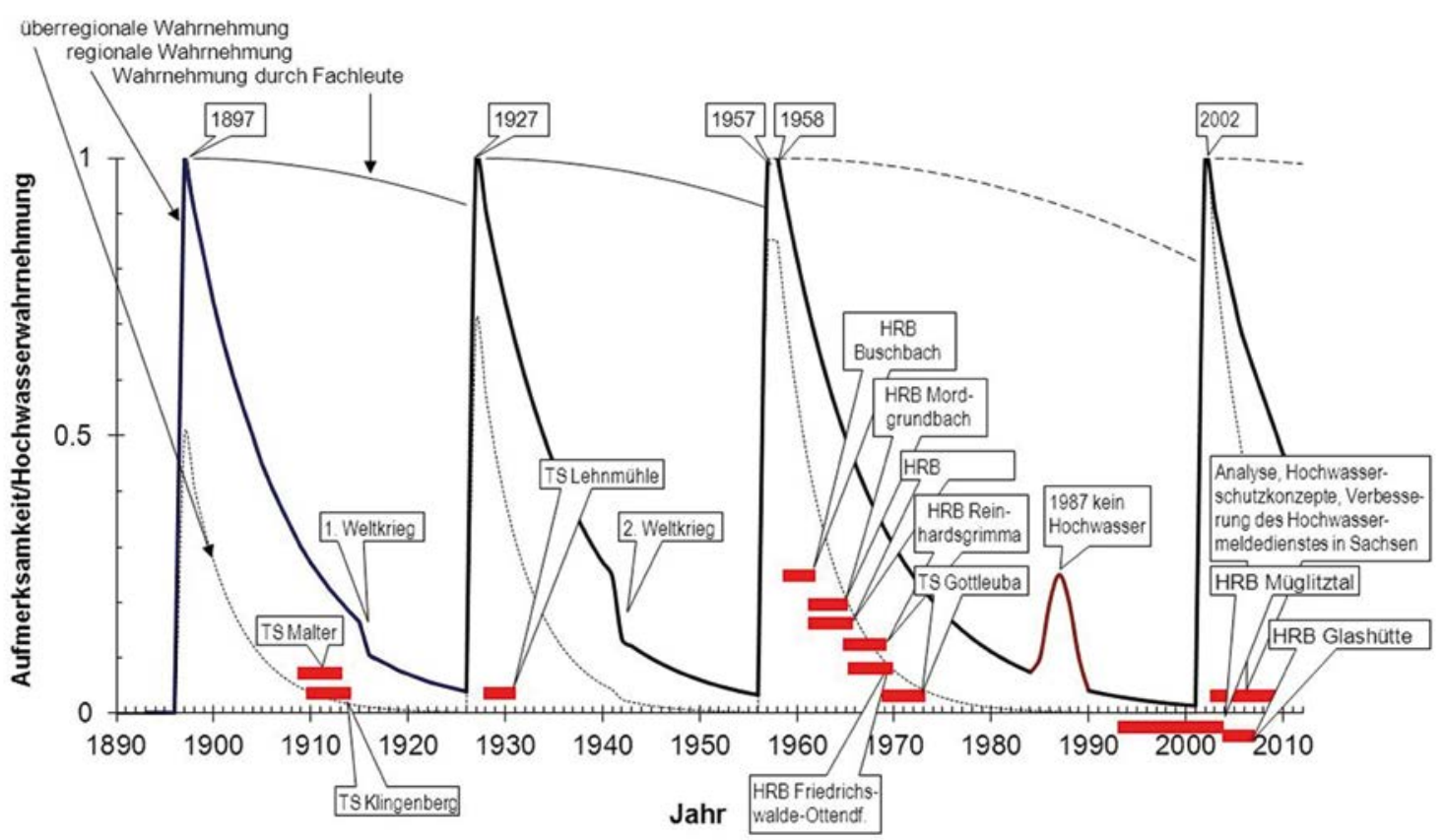

Bild 1: "Gefühltes“ empirisches Hochwasserbewusstsein im Osterzgebirge als Aufmerksamkeitskurve mit Maßnahmen der Vorsorge (ohne lokale, kleinsträumige Ereignisse) 


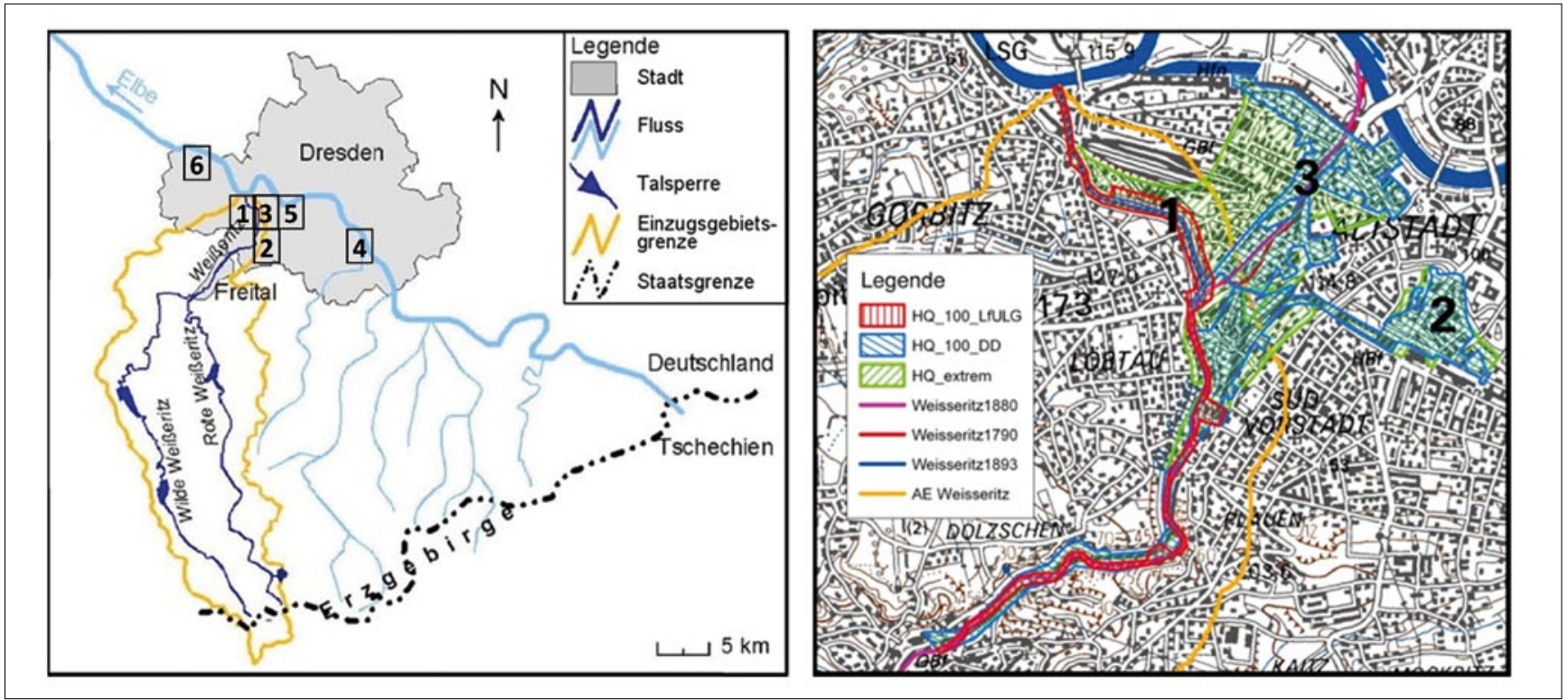

Bild 2: Gesamteinzugsgebiet der Weißeritz und Teileinzugsgebiet der sächsischen Elbe; Innenstadt von Dresden

Weißeritz, einem im Erzgebirge entspringenden linken Nebenfluss der Elbe überflutet. Der Scheiteldurchgang des Elbehochwassers am Dresdner Pegel erfolgte 5 Tage später.

Die Weißeritz entwässert ein Einzugsgebiet von $385 \mathrm{~km}^{2}$. Davon entfallen $162,7 \mathrm{~km}^{2}\left(150,3 \mathrm{~km}^{2}\right.$ auf deutscher Seite) auf das Einzugsgebiet der Wilden Weißeritz mit einer Fließstrecke von $47 \mathrm{~km}$. Die Rote Weißeritz hat ein Einzugsgebiet von $154,3 \mathrm{~km}^{2}$ mit einer Lauflänge von $35 \mathrm{~km}$.
Die restliche Fläche von $66,7 \mathrm{~km}^{2}$ mit einer Flusslänge von $13,7 \mathrm{~km}$ gehört zur Vereinigten Weißeritz. Von dieser befinden sich 8,2 km im Dresdner Stadtgebiet, wo die Weißeritz auch am Flusskilometer $61,5 \mathrm{~km}$ in die Elbe mündet. Die Weißeritzmündung war bis 1892 bei Elb-Kilometer 56,7 km an der Marienbrücke [4] und wurde damals an die jetzige Stelle verlegt. In Bild 2 sind die beiden Flussläufe und die Umfragegebiete dargestellt. Das Gebiet 1 beinhaltet die Wohngebiete

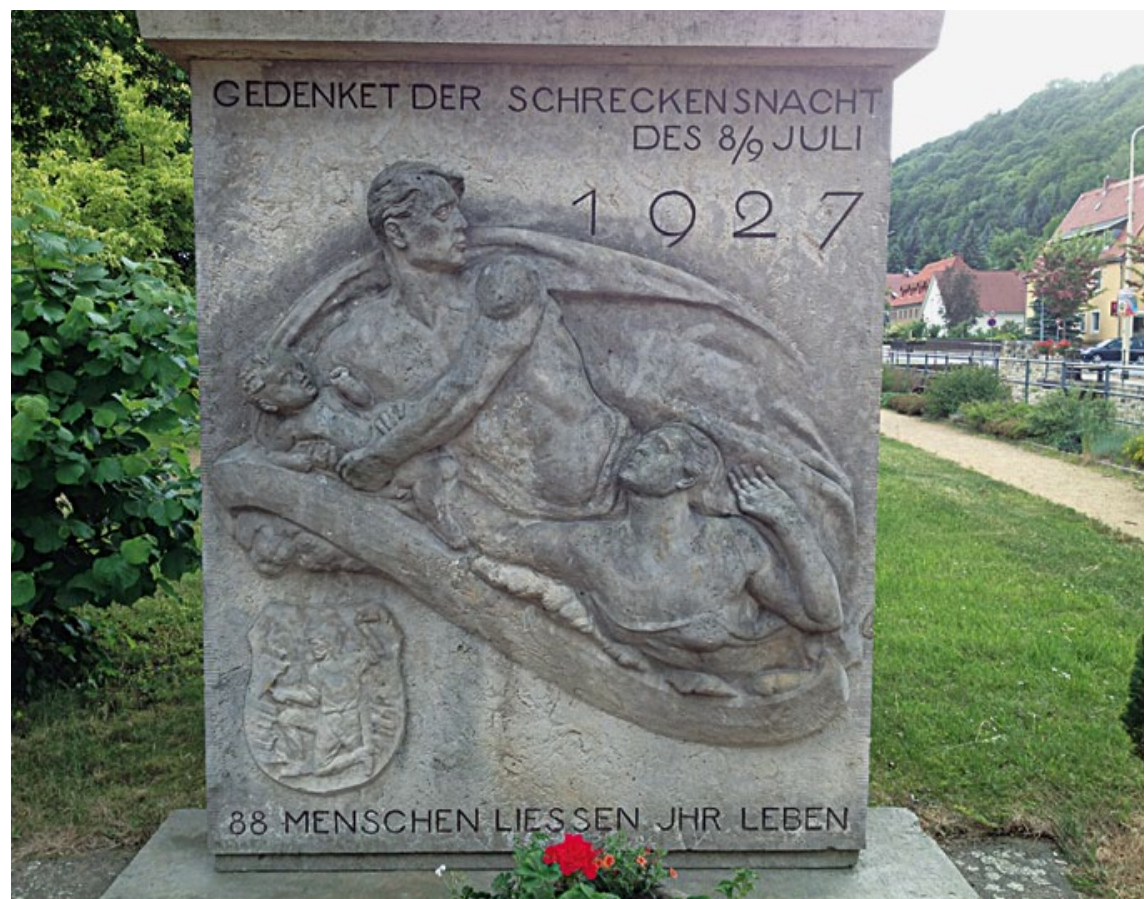

Bild 3: Denkmal für die Hochwasseropfer der Gottleuba von 1927 in Berggießhübel im Erzgebirge direkt am Weißeritzufer, das Gebiet 2 die Seevorstadt-Ost bzw. Großer Garten und das Gebiet 3 umfasst Teile der Friedrichstadt sowie der Wilsdruffer Vorstadt. Im Stadtgebiet von Dresden nimmt die Vereinigte Weißeritz ein Einzugsgebiet von $24 \mathrm{~km}^{2}$ ein, in dem etwa 60000 Einwohner leben, davon etwa 4700 im hundertjährlichen Überflutungsgebiet. Die Umfrage im vom Elbehochwasser betroffenen Gebiet wurde hauptsächlich in Laubegast (Gebiet 4) dem Stadtzentrum (5) und Stetzsch/Gohlis (6) durchgeführt (Bild 2).

Die sogenannte "Jahrhundertflut" 2002, deren Bezeichnung, wie schon früher gezeigt werden konnte [4], eher umgangssprachlich als wissenschaftlich zu verstehen ist, entstand aus einer Vb-Wetterlage mit extremen Niederschlägen u. a. im Erzgebirge. Am 12.08.2002 fiel im Erzgebirge und im südlichen Einzugsgebiet der Moldau so viel Regen wie sonst in drei Monaten. Dadurch stieg der Durchfluss vor allem in den kleinen Gebirgsflüssen wie der Roten und Wilden Weißeritz sowie der Müglitz, und einige Tage später auch in der Elbe stark an. Durch Geschiebe- und Treibguttransport kam es zu Ablagerungen und Verklausungen. Dies trug dazu bei, dass oberhalb der ersten Dresdner Weißeritzbrücke und am Weißeritzknick der Fluss am 12.08.2002 gegen 22:30 Uhr über die Ufer trat und sich im ehemaligen Flussbett entlang der Löbtauer und der Weißeritzstraße seinen Weg bahnte, wobei durchaus Fließgeschwindigkeiten von $5 \mathrm{~m} / \mathrm{s}$ auftraten. Ab der Nossener Brücke folgte 

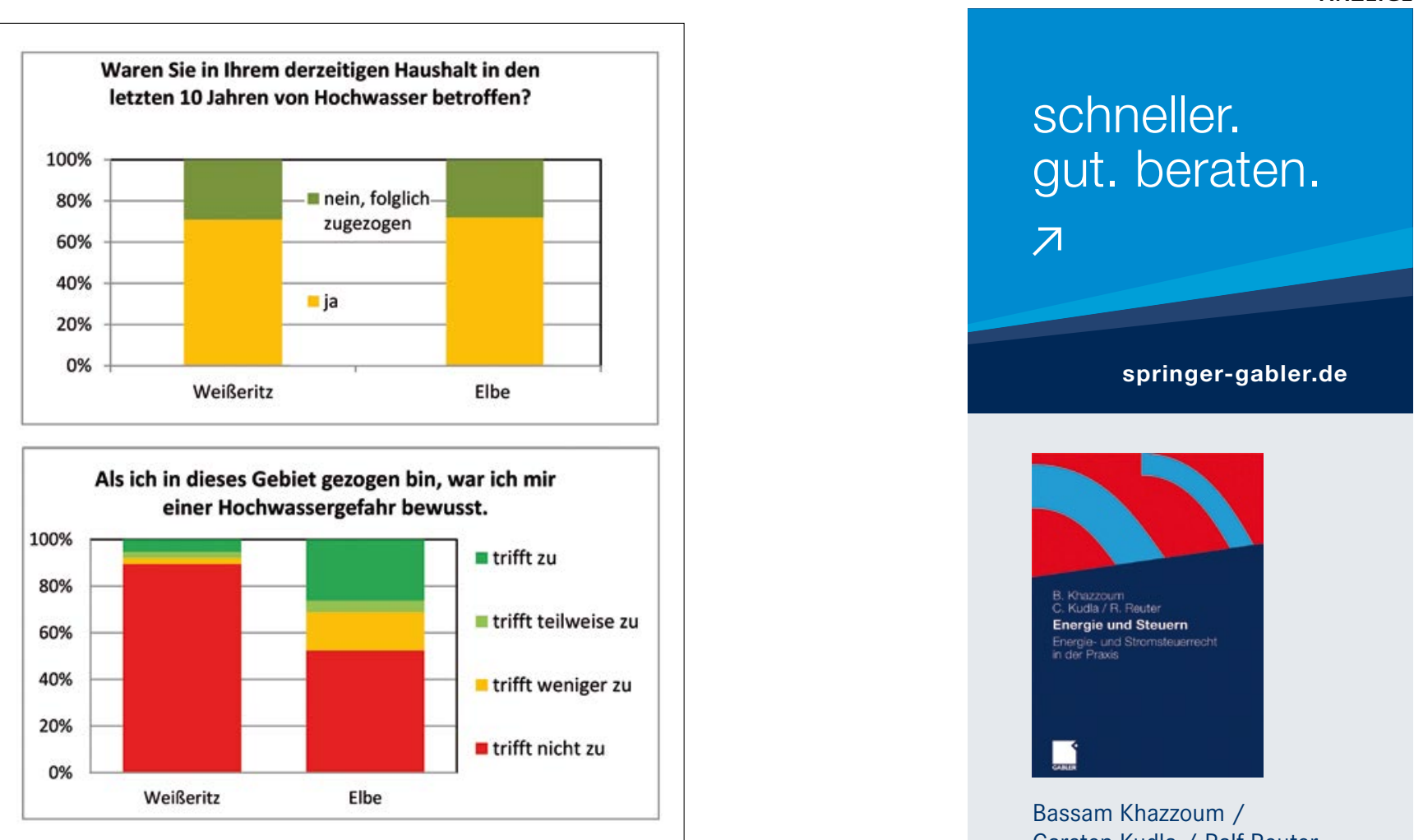

Bild 4: Erfahrung mit früheren Hochwasserereignissen

ein anderer Teil des Wassers der Weißeritz dem tiefer liegenden Eisenbahngleis zum Hauptbahnhof, überflutete diesen und angrenzende Stadtgebiete wie die Prager Straße. Vom Ausmaß, der Ausdehnung und auch nach dem Schadensbild war das Augusthochwasser 2002 mit den Ereignissen von 1897 oder $1927 \mathrm{zu}$ vergleichen. Letzterem gewidmet ist ein Denkmal in Berggießhübel/Gottleuba (Osterzgebirge), welches die Erinnerung wach und das Hochwasserbewusstsein hoch halten soll (Bild 3).

\subsection{Aufbau des Fragebogens}

Der Fragebogen setzte sich aus sechs Abschnitten zusammen:

- Abschnitt 1: Naturgefahren und Hochwasser allgemein (Frage 1 bis 9),

- Abschnitt 2: Hochwasserfolgen (Frage 10 bis 21),

- Abschnitt 3: Kommunikation und Information (Frage 22 bis 35),

- Abschnitt 4: Selbstschutz und Eigenvorsorge (Frage 36 bis 50),

- Abschnitt 5: Finanzielle Auswirkungen (Frage 51 bis 59) und

- Abschnitt 6: Fragen zur Person (Frage 60 bis 69).

\subsection{Durchführung der Umfrage}

Es war beabsichtigt, in den untersuchten Gebieten jeden vierten Haushalt zu befragen. Die Verteilung der Fragebögen erfolgte teils persönlich, teils als Postwurfsendung. Die Rückgabe erfolgte per Post, per Fax oder durch Abholung.

Von den knapp 4000 Haushalten (Weißeritz $1901+$ Elbe 2 010) in den ausgewählten Überschwemmungsgebieten wurden 1008 (Weißeritz 666 + Elbe 342) befragt, von denen 372 (Weißeritz 238 + Elbe 134) antworteten, was knapp $10 \%$ der Haushalte im entsprechenden Gebiet entsprach [3], [6], [7]. Bei einer Vertrauenswahrscheinlichkeit von $95 \%$ und einer zulässigen Abweichung der relativen Häufigkeit vom wahren Wert

Bassam Khazzoum / Carsten Kudla / Ralf Reuter

Energie und Steuern Energie- und Stromsteuerrecht in der Praxis

2011. 184 S. Br. EUR 49,95 ISBN 978-3-8349-2272-4

Die Energiesteuer ist als Verbrauchsteuer eine indirekte Steuer mit zahlreichen Besonderheiten bei den Steuertarifen, Steuerbegünstigungen, Steuerbefreiungen und -ermäßigungen. Das Werk erläutert diese und gibt fundierte Hinweise für die steueroptimale Anwendung in der Praxis.

Das Gesetz zur Änderung des Energiesteuer- und des Stromsteuergesetzes ist bereits eingearbeitet.

Einfach bestellen:

SpringerDE-service@springer.com Telefon +49(0)6221/345- 4301 


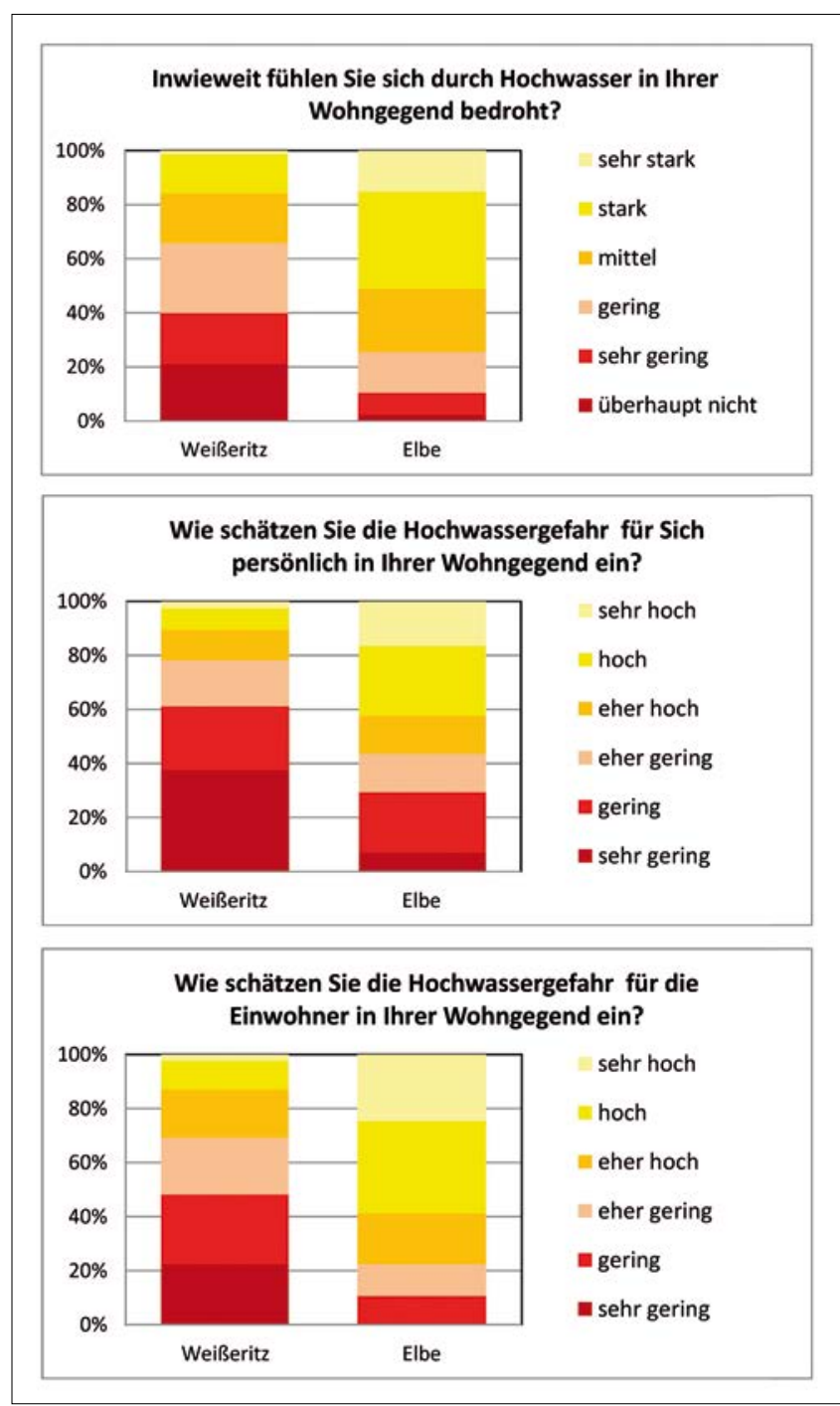

Bild 5: Gefährdung durch Hochwasser
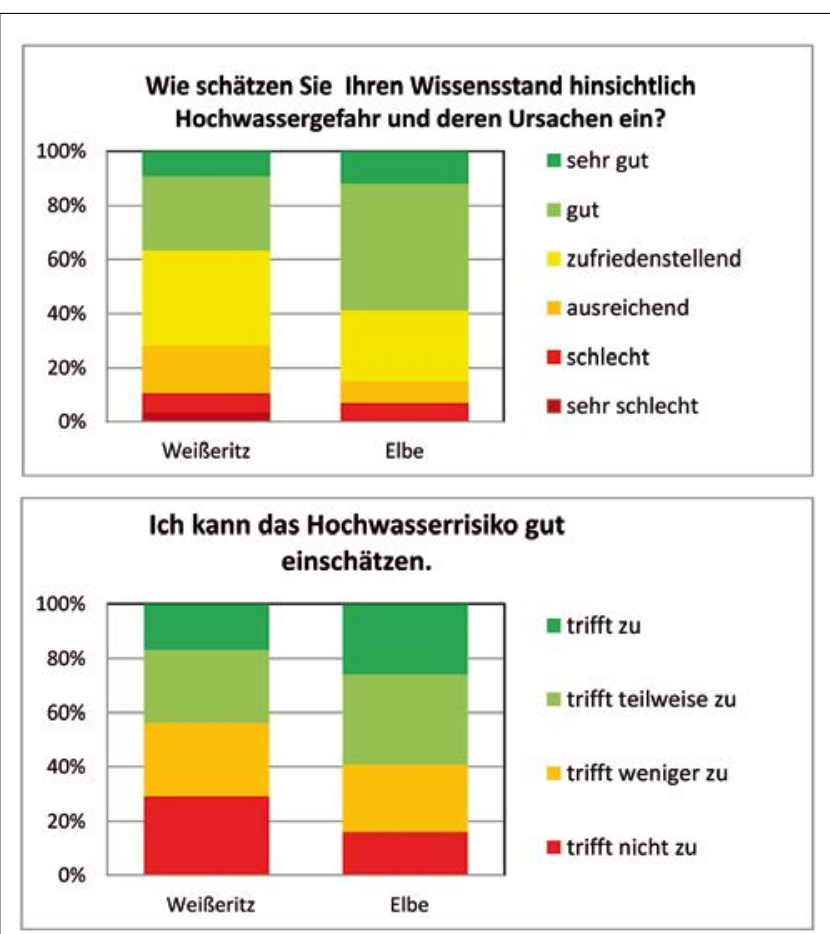

Ich bin mit der Hochwasserthematik vertraut.

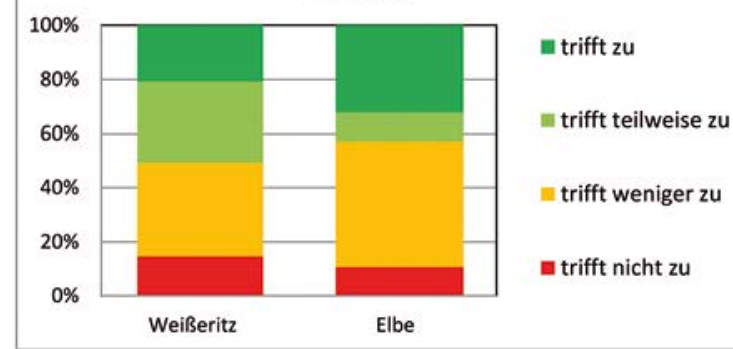

Bild 6: Hochwasserkenntnisse um 10 \% wären nur 96 Antworten erforderlich gewesen. Dies bedeutet, dass der relative Fehler der Antworthäufigkeiten für das Weißeritzgebiet $6 \%$ und für das 2002 an der Elbe überflutete Gebiet $8,3 \%$ beträgt [5].

Für die Nichtteilnahme an der Umfrage hatten die Einwohner verschiedene Gründe: kein Interesse, zwecklos, nicht betroffen, gesundheitlich nicht in der Lage, keine Zeit, überfordert, unerfahren, schon zu viele Umfragen, Verständnisprobleme (Personen mit Migrationshintergrund), Fragen zu persönlich, neu zugezogen oder andere Gründe.

\section{Umfrageergebnisse}

Die Auswertung im Rahmen der vorliegenden Untersuchung erfolgte für die Fragen, die Rückschlüsse auf das Hochwas- serbewusstsein erlauben. Diese waren vorwiegend in den Abschnitten 1, 2 und 4 des Fragebogens zu finden. Die Fragebögen wurden pro Haushalt beantwortet.

\subsection{Historie}

Da alle befragten Haushalte im Überschwemmungsgebiet von 2002 liegen, dadurch während des Hochwassers damals die Erreichbarkeit erschwert war und zudem auch die Bewohner oberer Etagen in der Regel über einen Kelleranteil oder einen Fahrzeugabstellplatz verfügen, hätte jeder Haushalt vom Hochwasser betroffen sein müssen.

Der in den Überschwemmungsgebieten von Elbe und Weißeritz annähernd gleich große nicht betroffene Teil von $30 \%$ zeigt demzufolge ungefähr den Anteil von Neuzuzügen in den letzten 10 Jahren zwischen dem Hochwasser und der Umfrage (Bild 4).

\subsection{Subjektive Gefährdung}

Die Frage nach der Hochwassergefahr in der Wohngegend erlaubte eine Unterscheidung nach der Gefahr für die Befragten, für die Bewohner des Hauses, für die Bewohner der Wohngegend und für die gesamte Stadt.

Es gab sechs Abstufungen von sehr hoch (1) bis sehr gering (6). Interessanterweise schätzten die Befragten die Gefahr für die Stadt und die Wohngegend größer ein als für sich selbst (Bild 5). Wie einige der Befragten erzählten, waren 2002, wenn überhaupt, nur die Familien im Erdgeschoss der mehrstöckigen Wohnhäuser vom Hochwasser direkt betroffen. Meist waren nur die Keller voll Wasser gelaufen.

Während sich im Überflutungsbereich der Elbe etwas mehr als die Hälfte der Teilnehmer überdurchschnittlich betroffen fühlen, sind es bei der deutlich kleineren Weißeritz weniger als $30 \%$. 
Offensichtlich ist das Hochwasserbewusstsein bei den Elbanrainern signifikant größer als bei denjenigen am Gebirgsfluss Weißeritz, bei dem zudem viele kleinere und mittlere Hochwasserereignisse durch die Retention der Talsperren im Oberlauf gar nicht mehr wahrgenommen werden (können).

\subsection{Kenntnisse}

Die Beschäftigung mit Naturgefahren und der Erwerb gewisser Grundkenntnisse sind eine Voraussetzung, um Vorsorge zu betreiben, Schutzmaßnahmen zu initiieren oder daran mitzuwirken und richtige Schlussfolgerungen im Gefahrenfall zu ziehen.

Entsprechend ihrer Selbsteinschätzung sind die Kenntnisse in Bezug auf Hochwasser und dessen Ursachen bei über $80 \%$ der Elbeanwohner mittel bis gut, während dies an der Weißeritz immerhin noch $70 \%$ der Befragten meinten. Etwa die Hälfte der Befragten kann nach eigener Einschätzung das Hochwasserrisiko zumindest teilweise gut einschätzen und ist mit der Thematik ganz oder teilweise vertraut (Bild 6).

\subsection{Hochwasserschutzmaßnahmen}

Um operativen Hochwasserschutz zu betreiben, ist eine zuverlässige Vorhersage erforderlich. Die Bewohner im Überflutungsgebiet wurden gefragt, wie gut ihrer Meinung nach die derzeitige Möglichkeit der Vorhersage von Überschwemmungen in ihrem Stadtteil ist. Wie bei zahlreichen Fragen gibt es auch hier eine Abstufung von sechs Kategorien von „sehr gut“ (1) bis „sehr schlecht“ (6). Im Kreisdiagramm Bild 7 ist zu erkennen, dass circa zwei Drittel der Einwohner die momentane Vorhersage von Überschwemmungen in ihrem Stadtteil tendenziell als gut einschätzen, was auf Vertrauen in die Arbeit zuständigen Fachleute schließen lässt.

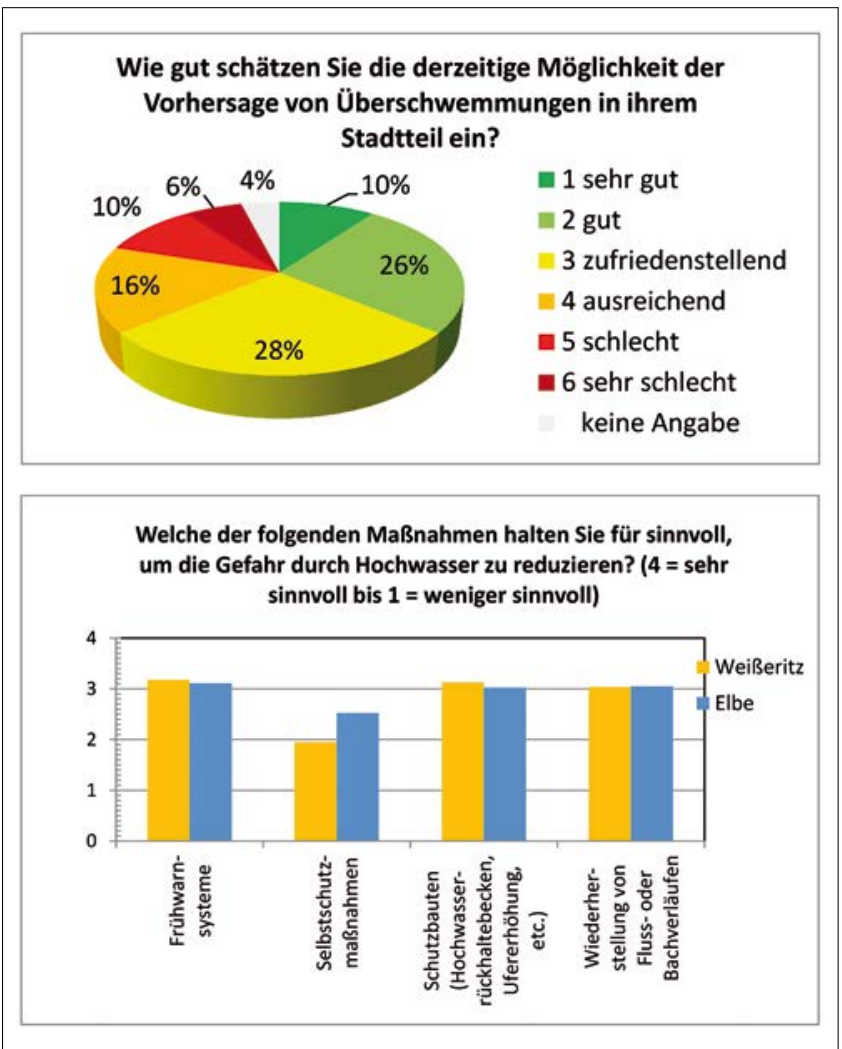

Bild 7: Hochwasservorhersage im Stadtteil und Schutzmaßnahmen
Aufschlussreich ist, dass bei der Frage nach der Sinnhaftigkeit möglicher Schutzmaßnahmen der Selbstschutz deutlich geringere Werte erzielt als Frühwarnsysteme, Schutzbauten und Renaturierung, obgleich die Hälfte (Elbe) oder ein knappes Drittel (Weißeritz) die Selbstschutzmaßnahmen zumindest kennt. Im Elbegebiet der Stadt wurden solche Maßnahmen auch von knapp $40 \%$ beim letzten Hochwasser angewandt, während es an der Weißeritz nur knapp 20 \% waren. Diejenigen, die keine Selbstschutzmaßnahmen angewandt hatten, meinten zu $90 \%$, dass diese nicht notwendig gewesen seien oder z. B. aus Gründen des zeitlichen Vorlaufes nicht möglich waren (Bild 8).

\section{Schlussfolgerungen und Ausblick}

Im vorliegenden Beitrag wurde versucht, eine Momentaufnahme des Hochwasserbewusstseins der Einwohner in ausgewählten Überflutungsgebieten des Sommerhochwassers 2002 nachzuzei-

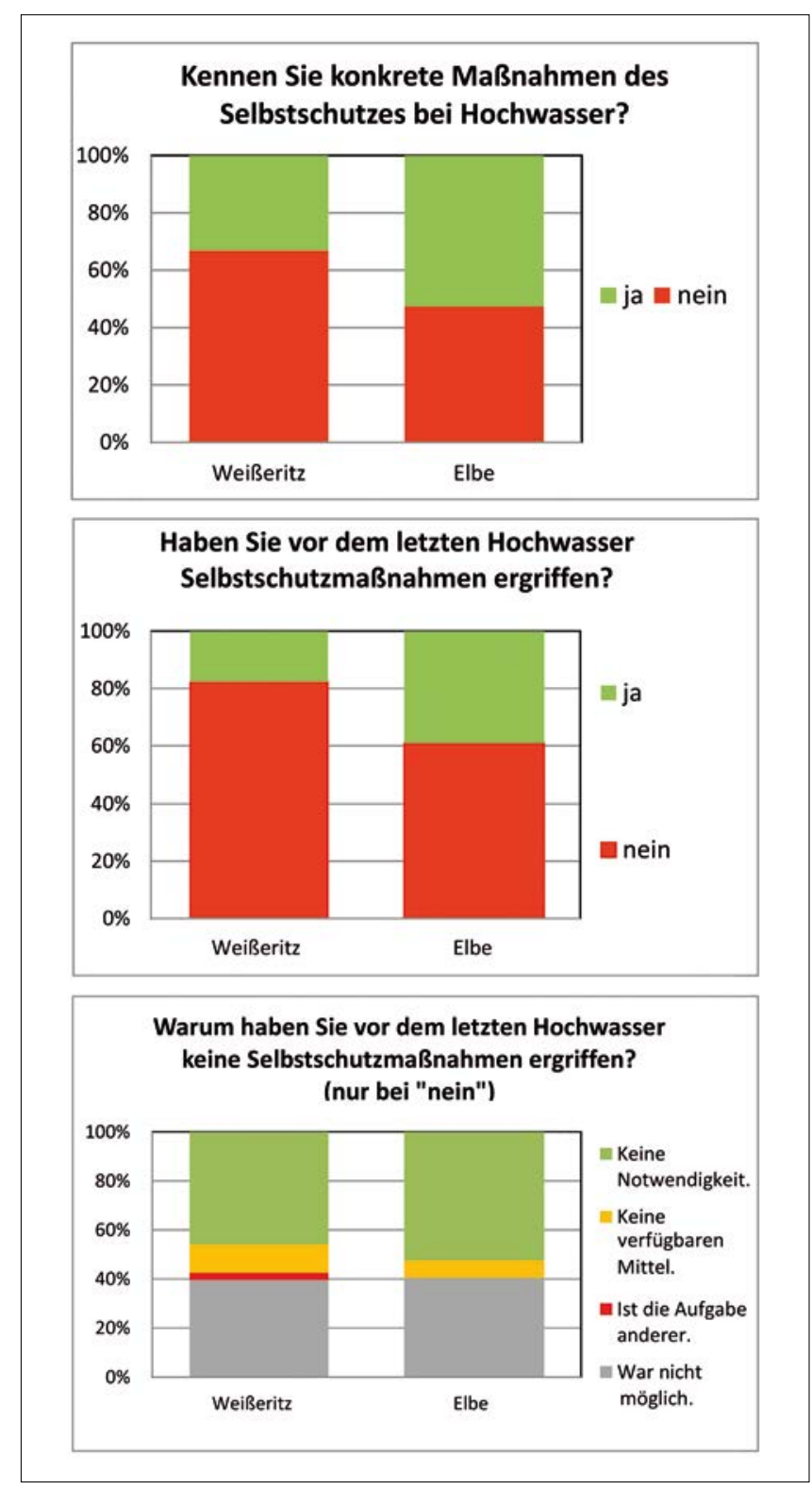

Bild 8: Hochwasserschutzmaßnahmen 
chnen. Die statistische Auswertung von Umfrageergebnissen hat gezeigt, dass das Hochwasserbewusstsein der in den Überflutungsgebieten von 2002 lebenden Befragten nur einen mittleren Stand repräsentiert, den man näherungsweise mit einer auf $50 \%$ gefallenen Aufmerksamkeitskurve veranschaulichen könnte.

$\mathrm{Zu}$ denken gibt die Tatsache, dass Selbstschutzmaßnahmen eine geringere Priorität eingeräumt wird als öffentlich organisierten und finanzierten Maßnahmen. Dieses Ergebnis könnte als Anhaltspunkt für eine zu gering ausgeprägte Eigenverantwortung gesehen werden, die zukünftig gestärkt werden sollte.

Beim Vergleich der Antworten aus den Überflutungsbereichen der Elbe und der Weißeritz, wo die möglichen Vorhersagezeiten wesentlich kürzer sind, fiel auf, dass trotz ansonsten objektiv ähnlicher Exposition die Befragung im Weißeritzgebiet ein geringeres Hochwasserbewusstsein als an der Elbe ergab. Möglicherweise liegt dies daran, dass für den wesentlich größeren Elbestrom auch ein wesentlich größeres Schadenspotenzial vermutet wird und dass die an den Weißeritztalsperren vorhandenen Rückhalteräume die kleinen sowie mittleren Hochwasserwellen aufnehmen und so die natürlichen Abflussschwankungen für die Unterlieger weniger erlebbar machen.

Für nachfolgende Untersuchungen wäre eine Verschneidung der Befragungsergebnisse mit Zensusdaten und Schadenspotenzialen vorteilhaft, um weitere $\mathrm{Zu}$ sammenhänge erkennen zu können.

In jedem Fall wird aus der Studie deutlich, dass das gegenwärtige individuelle Hochwasserbewusstsein nicht ausreicht, um auf ein mögliches zukünftiges Ereignis gut vorbereitet zu sein. Hier ist es notwendig, auch weiterhin Information und Öffentlichkeitsarbeit zur Entstehung, zum Ablauf und zu den Auswirkungen von Hochwasserereignissen zu betreiben und an der Verbesserung oder Erhaltung

Antje Bornschein and Reinhard Pohl

\section{Flood Awareness 10 Years after the „Flood of the Century" in the Eastern Erzgebirge Mountains and at the Elbe River}

Since the extreme flood in Saxony in 2002 much has been done for flood protection during the last decade: prediction, communication and flood protection have been improved. New levees, protection works and flood retaining reservoirs have been built or upgraded. But a very important question is how the flood awareness of the people at risk has developed meanwhile. This paper investigates whether the lessons learnt in 2002 have been handed down and are still in mind or the awareness has flagged and people are lulling themself into a false sense of safety because of the improved protection.

\section{Антье Борншайн и Райнхард Поль}

\section{«Паводковое сознание»: через 10 лет после «наводнения века» в восточном регионе Эрцгебирге и на Эльбе}

В течение 10 лет, прошедших после катастрофического наводнения в Саксонии, было много сделано в области противопаводковой защиты:улучшены методы прогнозирования и способы коммуникации, усовершенствованы противопаводковые сооружения, возведены новые дамбы, а также построены или усовершенствованы водохранилища паводкового регулирования. При том, однако, возник важный вопрос о том, насколько «паводковое сознание» развилось у потенциально затронутых групп населения. В данной статье рассматриваются следующие аспекты: остался ли в памяти урок 2002 года и передается ли опыт дальше или же «паводковое сознание» ослабло и население считает себя в безопасности благодаря улучшению противопаводковой защиты. des Hochwasserbewusstseins als Daueraufgabe zu arbeiten, auch durch die Erhaltung von Hochwassermarken und -denkmälern.

Dank

Teile der vorliegenden Arbeit sind im $\mathrm{Zu}$ sammenhang mit dem EU-Projekt „Sustainable Strategies of Urban Flood Risk Management with non-structural Measures to cope with the Residual Risk" (SUFRI) im Rahmen des ERA-Net-CRUEProgramms entstanden. Die Autoren danken den Verwaltungsstellen der teilnehmenden EU-Länder für die Ermöglichung der Untersuchungen sowie Prof. Zenz und Ass. Prof. Knoblauch von der TU Graz für die Unterstützung und sehr gute Zusammenarbeit.

\section{Autoren \\ Dr.- Ing. Antje Bornschein \\ Prof. Dr.-Ing. habil. Reinhard Pohl \\ Institut für Wasserbau und Technische \\ Hydromechanik der TU Dresden \\ 01062 Dresden \\ Antje.Bornschein@tu-dresden.de \\ Reinhard.Pohl@tu-dresden.de}

\section{Literatur}

[1] Merz, B; Bittner, R; Grünewald, U.; Piroth, K. (Hrsg.): Management von Hochwasserrisiken. Stuttgart: Schweizerbart Science Publishers, 2011.

[2] Pareto, V.: Cours d'économie politique professé à l'Université de Lausanne. , Lausanne: $\mathrm{F}$. Rouge 1896/1897 (2 volumes)

[3] Poersch, N.: Quantifizierung von Hochwasserrisiken und Hochwasserrezeption gesternheute-morgen. Diplomarbeit am Institut für Hydrologie und Meteorologie der TU Dresden, 2011.

[4] Pohl, R.: Historische Hochwasser aus dem Erzgebirge. In: Dresdner Wasserbauliche Mitteilungen (2004), Heft 28.

[5] Sachs, L.: Angewandte Statistik. Berlin, Heidelberg, New York: Springer Verlag, 1992.

[6] Schmidt, A. (2011): Automatische Hochwasserfrühwarnsysteme für kleine Einzugsgebiete. Diplomarbeit, Institut für Hydrologie und Meteorologie, TU Dresden

[7] Sieckmann, M.-Ch.: Hochwassergefahren und deren Abwehr in urbanen Gebieten. Diplomarbeit am Institut für Hydrologie und Meteorologie der TU Dresden, 2011.

[8] Walther, P.; Pohl, R.: Hochwasserrisiken im Osterzgebirge - eine historische Retrospektive. In: Dresdner Wasserbauliche Mitteilungen (2004), Heft 27.

[9] Abschlussbericht des ERANET-CRUE-Projektes "Sustainable Strategies of Urban Flood Risk Management with non-structural Measures to cope with the Residual Risk" SUFRI, 2011 (www.sufri.tugraz.at). 